\title{
EVALUACION DE UN ALGORITMO DE TORQUE VECTORING CON CAPACIDAD DE FRENADO REGENERATIVO
}

\author{
Alberto Parra ${ }^{a}$, Asier Zubizarreta ${ }^{b}$, Joshué Pérez ${ }^{a}$. \\ ${ }^{a}$ Tecnalia Research \& Innovation, División de Industria y Transporte, España \\ ${ }^{b}$ Departamento de Ingeniería de Sistemas y Automática, Universidad del País Vasco (UPV/EHU)
}

Resumen Facultad de Ingeniería de Bilbao, España

Los sistemas inteligentes de transporte (ITS) son actualmente una de las áreas de investigación más activas, siendo los vehículos eléctricos (EV) y la mejora de su comportamiento dinámico temas clave. Para este propósito, es necesario el desarrollo de sistemas avanzados de asistencia a la conducción (ADAS) y sistemas avanzados de control para la dinámica vehicular. Convencionalmente, estos sistemas se han centrado en aumentar la estabilidad del vehículo en escenarios críticos. Sin embargo, los vehículos eléctricos permiten incluir también la eficiencia, haciendo uso del frenado regenerativo como una variable de control. Para poder diseñar sistemas de control tan sofisticados, es necesario implementar técnicas de control capaces de gestionar tanto la estabilidad como la eficiencia. En este sentido, las técnicas de control inteligente han demostrado ser una de las mejores alternativas. En este trabajo se presenta un algoritmo de distribución de par, Torque Vectoring (TV), basado en técnicas de control inteligente y con capacidades de frenado regenerativo. El algoritmo de TV presentado ha sido implementado en un sistema embebido y validado en un entorno de "Hardware in the Loop" (HiL). Los resultados muestran que el sistema presentado no solo es capaz de mejorar el comportamiento dinámico del vehículo en una maniobra de emergencia desafiante, sino también aumentar su eficiencia.

Palabras clave: Vehículo eléctrico, Torque Vectoring, dinámica vehicular, frenada regenerativa.

\section{INTRODUCCIÓN}

El desarrollo de sistemas de transporte de baja contaminación, eficientes y seguros es uno de los principales desafíos para la sociedad. El interés de los vehículos con sistemas de propulsión eléctrica (totalmente eléctricos e híbridos) ha aumentado en los últimos años, convirtiéndose en una de las principales áreas de investigación en la industria automotriz. Estos sistemas de propulsión pueden implementarse en una amplia gama de topologías, desde un solo motor eléctrico con un por rueda. El uso de topologías de varios motores permite mejoras significativas no solo en términos de eficiencia y aprovechamiento del espacio, sino también en términos de comportamiento dinámico del vehículo [10]. De esta manera, con los sistemas de control adecuados, se puede mejorar el confort, la eficiencia y la seguridad del vehículo [20].

Los sistemas avanzados de asistencia a la conducción (ADAS) y los sistemas avanzados de control de dinámica vehicular son fundamentales para lograr este objetivo. Las soluciones mas comunes implementadas en vehículos comerciales están basadas en sistemas de tracción (TCS), sistemas de antibloqueo de ruedas o en sistemas de distribución de par (TV) [3]. Sin embargo, para el caso particular de los vehículos eléctricos con sistemas de propulsión con varios motores, las soluciones basadas en algoritmos de TV presentan un mayor potencial. A través de este sistema, es posible controlar el par de cada motor eléctrico individualmente, y por lo tanto, de cada rueda, siendo posible controlar el momento en el eje vertical del vehículo, el cual está directamente relacionado con la estabilidad del vehículo [6].

Para implementar un sistema de TV adecuado, se requiere un generador de referencia de velocidad angular en el eje vertical (yaw rate) óptimo. Esto generalmente se lleva a cabo considerando el nivel deseado de subviraje [19] [18] y/o incluyendo consideraciones de eficiencia energética [20]. La referencia de yaw rate generada es, entonces, comparada con la medida actual (fácilmente medible con una plataforma inercial), para calcular el momento deseado en el eje vertical del vehículo [3]. Se debe tener en cuenta que el ángulo de deslizamiento lateral del vehículo, que se puede medir o estimar [22], generalmente también se considera en los trabajos de TV [21].

Además, las fuerzas de los neumáticos también deben considerarse en los sistemas de TV, ya que indican qué neumático tiene más carga $\mathrm{y}$, por lo tanto, más agarre, siendo un elemento crucial en la dinámica del vehículo. Su medición o estimación es un factor clave para el diseño de los sistemas de $\mathrm{TV}$, ya que afecta directamente a la respuesta en 
las curvas del vehículo. Por lo tanto, esta variable es importante para lograr una distribución de par óptima. Entre las soluciones propuestas para estimar las fuerzas de los neumáticos, la más común es el uso de estimadores basados en modelo, como el modelo de neumático lineal [16], el modelo de Dugoffs [17], o modelos semiempíricos como el de Pacejka [5]. También se han propuesto sistemas de modelado a través de técnicas de control inteligente, que reducen la necesidad de conocer la compleja dinámica de los neumáticos [27].

Finalmente, el algoritmo de TV se puede basar en una amplia variedad de técnicas de control, desde los sistemas tradicionales hasta los más complejos. Los primeros, que tienen una formulación mas simple, como los basados en controladores Proporcionales-Integrales-Derivativos (PID) [3], demuestran un buen desempeño en condiciones de estado estacionario. Por otro lado, tambíen existen soluciones basadas en técnicas de control más complejas, como aquellas que utilizan un controlador predictivo [14] o un controlador en modo deslizante (SMC) [11], proporcionando buenos resultados no solo en condiciones de estado estacionario, sino también en condiciones transitorias. Sin embargo, generalmente requieren un alto coste computacional y presentan una alta dependencia del modelo, lo que limita su rendimiento si no se realiza un modelado adecuado.

Los sistemas de control inteligente, como aquellos basados en redes neuronales [15] o en sistemas de lógica difusa [7], también se han considerado como una alternativa para implementar algoritmos de TV. Estos proporcionan no solo una menor dependencia del modelo, sino también un menor coste computacional y una mayor capacidad para controlar sistemas de alta complejidad. Además, si se quieren lograr múltiples objetivos, las técnicas de control inteligente, como la lógica difusa o los algoritmos genéticos, presentan más ventajas que los sistemas mencionados anteriormente [31].

En el caso de los vehículos eléctricos, la eficiencia es un tema clave a considerar para maximizar la autonomía del vehículo. Sin embargo, en la literatura, la mayoría de los trabajos se centran exclusivamente en mejorar el comportamiento dinámico y la estabilidad del vehículo. Por lo tanto, este es todavía un campo abierto de gran potencial para ser explorado.

Al ser la eficiencia una variable clave a considerar junto con la estabilidad en los trabajos de $\mathrm{TV}$, en este trabajo se presenta un sistema de TV basado en lógica difusa con capacidades de frenado regenerativo. El sistema desarrollado hace uso de un estimador de fuerzas verticales en los neumáticos, el cual se presentó en [12]. El cor- recto funcionamiento del sistema propuesto se ha validado a través de una maniobra de emergencia, comparando los resultados con otros dos escenarios: un vehículo con el mismo sistema de TV pero deshabilitando el frenado regenerativo; y un vehículo sin sistema de TV. Además, para cuantificar el efecto del sistema de TV propuesto en la eficiencia general del vehículo, se ha comparado la energía consumida durante una vuelta en tres circuitos profesionales (Nurburgring, Montmelo y Silverstone) se comparará para los diferentes escenarios. Por último, se ha escogido un vehículo de Clase C para las pruebas, y la simulación se llevará a cabo utilizando el simulador de dinámica vehicular Dynacar [4].

Para realizar este estudio, el resto del trabajo se divide de la siguiente manera. En la Sección 2 se explica el sistema de TV propuesto. La Sección 3 describe la metodología de validación seguida, donde se detalla la maniobra seleccionada y el modelo del vehículo. La sección 4 explica los resultados más importantes. Finalmente, las conclusiones se resumen en la sección 5 .

\section{ALGORITMO DE TORQUE VECTORING}

La estructura general del sistema de TV propuesto se muestra en la Figura 1. El sistema combina una estrategia de distribución de par lateral (la parte superior) y una estrategia de distribución de par longitudinal (parte inferior), que se combinan en el subsistema de frenado regenerativo para, finalmente, calcular el par que se aplicará a cada motor. Cada uno de estos sistemas de distribución se detallan a continuación.

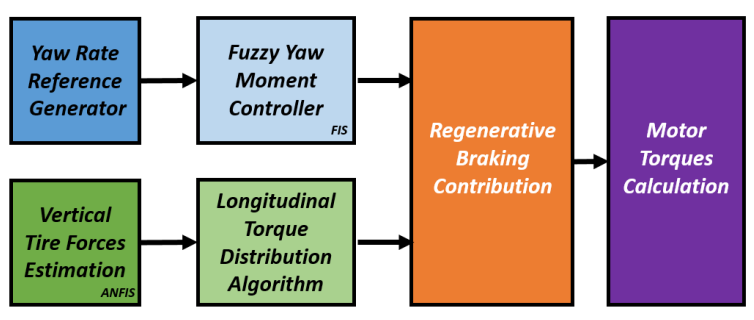

Figura 1: Estructura general del TV propuesto

\subsection{Distribución lateral de par}

El algoritmo de distribución lateral está compuesto por dos módulos: el generador de referencia de yaw rate y el controlador del momento del eje vertical del vehículo. Este último está basado en el que se presentó en [12] y calcula el porcentaje de par que se aplicará a cada lado del vehículo $\left(\tau_{\text {lat }}\right)$. Para ese propósito, el controlador requiere 
tres entradas (error de yaw rate, derivada del error de yaw rate y error de ángulo de deslizamiento lateral). El error de yaw rate se calcula como la resta entre el valor actual medido y la referencia generada. Esta referencia se calcula basándose en el conocido modelo de bicicleta, ya que proporciona un buen equilibrio entre precisión y coste computacional. De esta manera, la ecuación de referencia de yaw rate es [3]:

$$
\dot{\psi}_{\text {ref }}=\frac{V}{L+\frac{m}{L}\left(\frac{b}{C_{\alpha F}}-\frac{a}{C_{\alpha R}}\right) V^{2}} \delta
$$

donde $m$ es la masa total del vehículo, $a$ y $b$ son las distancias al centro de gravedad de los ejes delantero y trasero respectivamente, $L$ es la distancia entre ejes, $\delta$ es el ángulo de giro de las ruedas delanteras, $C_{\alpha F}$ y $C_{\alpha R}$ son los coeficientes de rigidez lateral de las ruedas delanteras y traseras, respectivamente, y $V$ es la velocidad del vehículo.

\subsection{Distribución longitudinal de par}

El objetivo del algoritmo de distribución de par longitudinal es destinar mayor par a las ruedas del eje que tiene mas agarre. Para ese propósito, se propone un algoritmo de distribución de par simple pero efectivo, basado en la máxima fuerza vertical que se puede aplicar en un eje (se ha tomado el delantero como referencia) y en la estimación de las fuerzas verticales en las ruedas de cada eje $f_{\text {wheels: }}$ :

$$
\tau_{\text {long }}=\frac{f_{\text {wheels }}}{f_{\max }}
$$

donde $f_{\text {wheels }}$ es la combinación de las fuerzas verticales de cada rueda de un eje (izquierda y derecha) y $f_{\max }$ es la fuerza normal máxima que se puede aplicar en el eje delantero (esto es, considerando que toda la masa está actuando sobre este eje).

Debe tenerse en cuenta que la medición de las fuerzas verticales de cada rueda no es una tarea trivial. En general, estas mediciones no son posibles y estos valores deben ser estimados. En este trabajo, se ha implementado el estimador neuroborroso basado en el propuesto en [12]. Este estimador requiere variables dinámicas del vehículo fácilmente medibles mediante una plataforma inercial y un GPS, y ha sido ampliamente validado mediante varias maniobras en [12], demostrando su correcto desempeño ( $3 \%$ de error medio).

\subsection{Contribución de frenada regenerativa}

Como se ha comentado en la sección 1, el sistema de frenado regenerativo no solo puede mejo- rar la eficiencia general del vehículo, sino también su comportamiento dinámico, proporcionando una capacidad de giro adicional que contribuye a la mejora del rendimiento en las curvas.

En ese sentido, el componente de frenado regenerativo implementado en el sistema Torque Vectoring desarrollado solo se activa cuando el ángulo de deslizamiento lateral del vehículo es superior a $10^{\circ}$, ya que es un indicador de comportamiento no deseado (subviraje o sobreviraje). Por lo tanto, el par de frenado se aplicará en las ruedas internas o externas, dependiendo de la distribución de par lateral proporcionada por el controlador de lógica difusa desarrollado. Es decir, este par negativo se aplicará en la rueda interior cuando el vehículo sufra subviraje, para ayudarlo a aumentar su capacidad de giro, y en caso de sobreviraje, el par de frenado se aplicará en la rueda exterior para evitar la posible pérdida de estabilidad.

Sin embargo, debe tenerse en cuenta que esta capacidad de giro adicional generada por el frenado regenerativo debe controlarse estrictamente limitando el par regenerativo, ya que pueden surgir posibles pérdidas de estabilidad. Además, estos límites deben ajustarse para cada eje $\left(\gamma_{\text {Front }} \mathrm{y}\right.$ $\gamma_{\text {Rear }}$, ya que ambos ejes (delantero y trasero) no frenan por igual.

\subsection{Cálculo de par motor}

Finalmente, el último subsistema calcula la consigna exacta de par motor que se aplicará a cada motor $\left(\tau_{i j}\right)$, según el par solicitado por el conductor con el acelerador $(\tau)$, la distribución longitudinal $\left(\tau_{\text {long }}\right)$, los porcentajes de distribución de par lateral $\left(\tau_{\text {lat }}\right)$, el factor de contribución de frenado regenerativo $\left(\gamma_{\text {Front }} \mathrm{y} \gamma_{\text {Rear }}\right)$ y el ángulo de deslizamiento lateral $(\beta)$,

$$
\begin{aligned}
& \alpha_{F L}= \begin{cases}-\max \left(\min \left(\tau_{\text {lat }},\left(1-\tau_{\text {lat }}\right)\right), \gamma_{\text {Front }}\right), & \text { si } \beta \geq 10 \\
\tau_{\text {lat }}, & \text { si no }\end{cases} \\
& \alpha_{F R}= \begin{cases}-\max \left(\min \left(\tau_{\text {lat }},\left(1-\tau_{\text {lat }}\right)\right), \gamma_{\text {Front }}\right), & \text { si } \beta \geq 10 \\
1-\tau_{\text {lat }}, & \text { si no }\end{cases} \\
& \alpha_{R L}= \begin{cases}-\max \left(\min \left(\tau_{\text {lat }},\left(1-\tau_{\text {lat }}\right)\right), \gamma_{\text {Rear }}\right), & \text { si } \beta \geq 10 \\
\tau_{\text {lat }}, & \text { si no }\end{cases}
\end{aligned}
$$

$$
\alpha_{R R}= \begin{cases}-\max \left(\min \left(\tau_{\text {lat }},\left(1-\tau_{\text {lat }}\right)\right), \gamma_{\text {Rear }}\right), & \text { si } \beta \geq 10 \\ 1-\tau_{\text {lat }}, & \text { si no }\end{cases}
$$




$$
\begin{aligned}
\tau_{f l} & =\tau \tau_{\text {long }} \alpha_{F L} \\
\tau_{f r} & =\tau \tau_{\text {long }} \alpha_{F R} \\
\tau_{r l} & =\tau\left(1-\tau_{\text {long }}\right) \alpha_{R L} \\
\tau_{r r} & =\tau\left(1-\tau_{\text {long }}\right) \alpha_{R R}
\end{aligned}
$$

\section{Metodología de validación}

En esta sección se explica la metodología de validación propuesta, incluido el vehículo seleccionado, el sistema embebido en el que se ha implementado, el modelo de simulación y la maniobra seleccionada.

Se pueden considerar tres sistemas principales. Primero, el modelo de conductor autónomo, que contiene un algoritmo de conducción automatizada que simula el comportamiento de un controlador estándar, garantizando que el rendimiento de cada prueba no depende del conductor. En segundo lugar, el bloque de control, que incluye el sistema de TV seleccionado (descrito en la sección 2). Finalmente, el modelo de vehículo, que simula la dinámica altamente no lineal del vehículo, usando Dynacar [4].

\subsection{Simulador de dinámica vehicular - Dynacar}

El modelo de vehículo se implementa en Dynacar, que es una plataforma de simulación de dinámica de vehículos de alta fidelidad desarrollada por Tecnalia Research \& Innovation [4]. Este software de simulación de vehículos ha sido validado a través de varias pruebas de pista [2].

El modelo físico del vehículo de Dynacar está basado en un modelo multicuerpo [1] e implementado en el entorno Matlab/Simulink para el desarrollo de sistemas de control para automoción. El tiempo de muestreo es de 1 milisegundo. Los neumáticos se modelan utilizando una implementación del modelo de Pacejka, ampliamente utilizado por la mayoría de los fabricantes de automóviles como un estándar de la industria para simulaciones orientadas a la dinámica vehicular [5].

En este trabajo, se ha seleccionado un vehículo de Clase C, cuyos parámetros principales se resumen en la Tabla 1. La topología del sistema de propulsión seleccionada para este trabajo es un sistema de tracción a las cuatro ruedas (AWD) con un motor controlado individualmente para cada rueda.

Teniendo en cuenta el vehículo mencionado anteriormente, las limitaciones para el par de frenado
Tabla 1: Parámetros principales del vehículo

\begin{tabular}{|c|l|}
\hline Masa $[\mathrm{kg}]$ & 1623 \\
\hline $\mathrm{Ix}\left[\mathrm{kg} \mathrm{m}^{2}\right]$ & 700 \\
\hline $\mathrm{Iy}\left[\mathrm{kg} \mathrm{m}^{2}\right]$ & 2300 \\
\hline $\mathrm{Iz}\left[\mathrm{kg} \mathrm{m}^{2}\right]$ & 2830 \\
\hline Dist. entre ejes $[\mathrm{m}]$ & 2.82 \\
\hline Anchura eje delantero $[\mathrm{m}]$ & 1.6 \\
\hline Anchura eje trasero $[\mathrm{m}]$ & 1.6 \\
\hline Radio del neumático $[\mathrm{m}]$ & 0.328 \\
\hline Dist. eje delantero al CG $[\mathrm{m}]$ & 1.5 \\
\hline Dist. eje trasero al CG $[\mathrm{m}]$ & 1.32 \\
\hline Altura del centro de gravedad $[\mathrm{m}]$ & 0.53 \\
\hline Área frontal $[\mathrm{m}]$ & 2.2 \\
\hline
\end{tabular}

regenerativo, se han establecido en un límite de par del $40 \%\left(\gamma_{\text {front }}\right)$ para el eje delantero y del $15 \%\left(\gamma_{\text {rear }}\right)$ para el eje trasero, con el objetivo de evitar pérdidas de estabilidad,

\subsection{Implementación del controlador}

El algoritmo de TV descrito en la Sección II se ha implementado en una plataforma embebida de Xilinx (Zynq XC7Z020), cuyas entradas y salidas se conectan mediante el bus CAN a un ordenador que ejecuta el simulador Dynacar y el modelo de conductor autónomo. Esto permite probar el rendimiento del sistema de TV propuesto en tiempo real, y no requiere del uso de un vehículo completo gracias al entorno HiL utilizado.

El sistema embebido seleccionado tiene un microcontrolador ARM que se ha utilizado para implementar el sistema de TV propuesto (Figura 1). Para ese propósito, el sistema desarrollado se implementó primero en Matlab Simulink y luego se compiló en código C para ejecutarlo en dicho microcontrolador con un requisito de tiempo de ciclo 10 milisegundos.

\subsection{Maniobra y escenarios seleccionados}

Para validar el sistema, se proponen dos escenarios: un primer escenario para evaluar el efecto del sistema propuesto desde el punto de vista de la estabilidad y el segundo para evaluar la eficiencia. Para el primer caso, se ha elegido la maniobra de doble cambio de carril [9], ya que es una maniobra muy desafiante para la dinámica del vehículo. Esta maniobra se detalla en la especificación ISO 3888 [9]. En esta prueba, el vehículo es llevado hasta cierta velocidad y se deja de actuar en el acelerador. Luego, el conductor intenta realizar la maniobra sin tocar los conos. La velocidad de la prueba se aumenta progresivamente hasta que se produce una inestabilidad o no se puede realizar la 
maniobra satisfactoriamente. Una maniobra tan exigente demuestra efectivamente la capacidad de giro de un vehículo cuando circula en el límite de tracción y, por lo tanto, esta prueba se considera una maniobra adecuada para evaluar los sistemas de control de dinámica vehicular por parte de muchos fabricantes de automóviles e instituciones de investigación.

Para este caso, es necesario realizar la prueba en condiciones de aceleración, ya que los algoritmos de TV actúan sobre el par de tracción. Para ello, se ha seleccionado una velocidad inicial de $50 \mathrm{~km} / \mathrm{h}$, y se ha aplicado un par constante a las ruedas de $2300 \mathrm{Nm}$ en total. Esto proporciona una aceleración longitudinal de 0,44 g, que permite obtener una velocidad final de $100 \mathrm{~km} / \mathrm{h}$, cubriendo el rango de velocidad más común de los vehículos de pasajeros en carreteras de velocidad media.

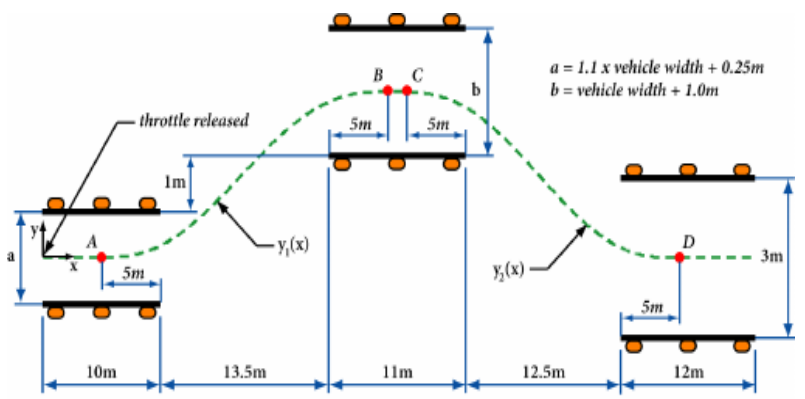

Figura 2: Doble cambio de carril

Para probar el rendimiento de eficiencia del enfoque de TV desarrollado, se define un segundo conjunto de escenarios, en los que se ha llevado a cabo una vuelta completa en los circuitos de Nurburgring, Montmeló y Silverstone, ya que incluyen una amplia variedad de circunstancias para un vehículo, en términos de combinaciones de aceleración/frenado y giro.

\section{Resultados}

Los resultados de la simulación para la maniobra de cambio doble de carril se muestran en las Figuras 3 - 6. La Figura 4 muestra la trayectoria seguida por el vehículo para cada caso. Como se puede ver en esta figura, la maniobra solo se completa con éxito ( $\sin$ golpear los conos) por el vehículo con el algoritmo de TV propuesto, reduciendo el subviraje sufrido en las otras configuraciones y, por lo tanto, demuestra una mejora en el manejo del vehículo en una maniobra de emergencia.

El efecto del algoritmo desarrollado se puede apreciar aún más en la Figura 5 en el intervalo de
$30 \mathrm{~m}$ a $40 \mathrm{~m}$ (marcado en verde). En esta parte de la maniobra, se puede ver la aceleración lateral adicional soportada por el vehículo y el yaw rate adicional generado por el par de frenado regenerativo aplicado en la rueda interior, ya que el vehículo tiende a sufrir subviraje en esta situación. Además, la Figura 6 muestra esta reducción de subviraje con la consecuente reducción del ángulo de deslizamiento de las ruedas delanteras, especialmente en el intervalo que se ha comentado, ya que esta es la parte más exigente de la maniobra. Más específicamente, la reducción del ángulo de deslizamiento es de aproximadamente $6^{\circ}$ en comparación con el vehículo sin algoritmo de TV y de $4^{\circ}$ en comparación con el vehículo con el algoritmo de TV pero sin la contribución de frenado regenerativo, lo que implica una reducción del $30 \%$ y $20 \%$, respectivamente.

Finalmente, la Figura 4 muestra la entrada del ángulo del volante proporcionada por el algoritmo de conductor autónomo. Se puede mostrar claramente que las variaciones en el ángulo de volante se reducen $\left(55^{\circ}\right.$ en comparación con el vehículo sin algoritmos de TV instalados y en $32^{\circ}$ en comparación con el vehículo con el algoritmo de TV pero sin la contribución de frenado regenerativo) en el intervalo ya comentado, demostrando la reducción del subviraje.

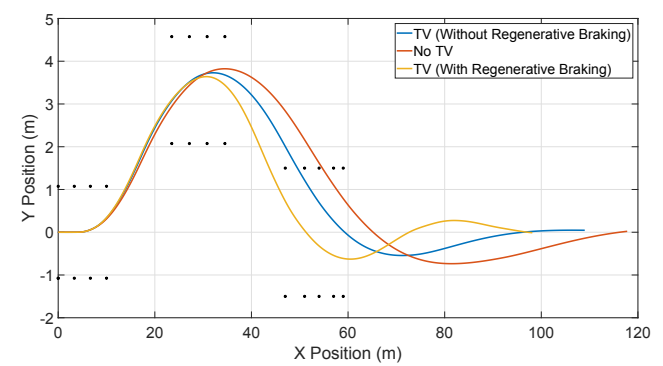

Figura 3: Doble cambio de carril: Trayectorias

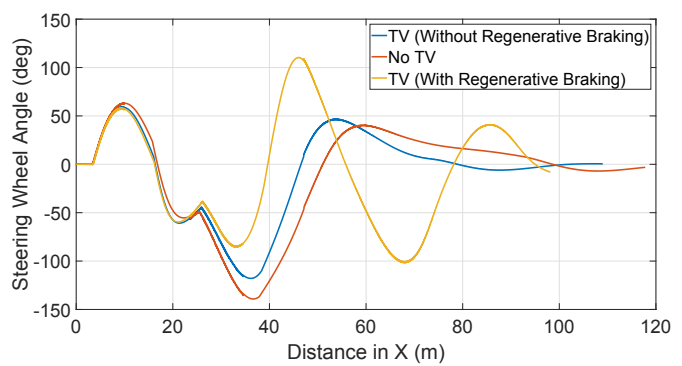

Figura 4: Doble cambio de carril: Ángulo de dirección

El efecto de cada algoritmo de TV en la eficiencia se puede apreciar notablemente en la Tabla 2 y en la Tabla 3, donde se muestra la energía 
Tabla 2: Comparativa de la energía eléctrica consumida

\begin{tabular}{|l|c|c|c|c|c|}
\cline { 2 - 6 } \multicolumn{1}{c|}{} & Sin TV & \multicolumn{2}{c|}{ TV } & \multicolumn{2}{c|}{ Regen TV } \\
\cline { 2 - 6 } \multicolumn{1}{c|}{} & Energía [kWh] & Energía [kWh] & Mejora [\%] & Energía [kWh] & Mejora [\%] \\
\hline Nurburgring & 17.01 & 14.04 & 17.46 & 13.19 & 22.45 \\
\hline Montmelo & 5.62 & 4.73 & 15.83 & 4.11 & 26.86 \\
\hline Silverstone & 8.34 & 6.78 & 18.70 & 6.20 & 25.65 \\
\hline
\end{tabular}

Tabla 3: Comparativa de la eficiencia

\begin{tabular}{|l|c|c|c|c|c|}
\cline { 2 - 6 } \multicolumn{1}{c|}{} & Sin TV & \multicolumn{2}{c|}{ TV } & \multicolumn{2}{c|}{ Regen TV } \\
\cline { 2 - 6 } \multicolumn{1}{c|}{} & Eficiencia [\%] & Eficiencia [\%] & Mejora [\%] & Eficiencia [\%] & Mejora [\%] \\
\hline Nurburgring & 76.70 & 77.17 & 0.47 & 84.94 & 8.24 \\
\hline Montmelo & 75.54 & 76.91 & 1.37 & 83.25 & 7.71 \\
\hline Silverstone & 76.31 & 78.13 & 1.82 & 83.77 & 7.46 \\
\hline
\end{tabular}
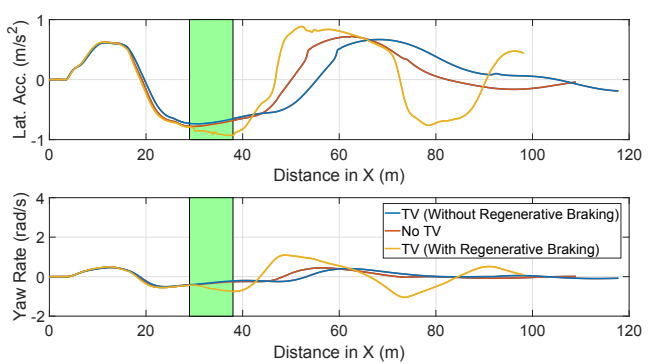

Figura 5: Doble cambio de carril: Aceleración Lateral y Yaw Rate
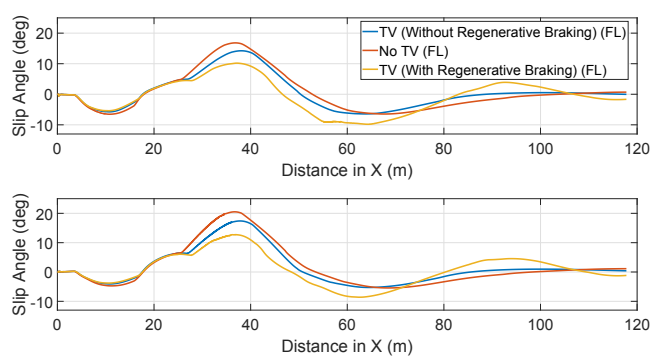

Figura 6: Doble cambio de carril: Ángulo de deslizamiento lateral de los neumáticos

mecánica consumida y la eficiencia general durante una vuelta completa en tres circuitos (Nurburgring, Montmelo y Silverstone) ejecutada por el algoritmo de conductor autónomo, configurado en modo agresivo. Los resultados demuestran que el sistema de TV propuesto proporciona un menor consumo de energía gracias a la contribución de frenado regenerativo con la consecuente mejora de la eficiencia. Debe tenerse en cuenta que el frenado regenerativo no se activa en cada condición de frenado, sino solo cuando el ángulo del deslizamiento es mayor de $10^{\circ}$, como ya se explicó en la Sección 2.

\section{Conclusiones}

El desarrollo de sistemas avanzados de ayuda a la conducción es un tema clave para el desarrollo de vehículos eléctricos con motores independientes en las ruedas. Convencionalmente, este tipo de sistemas tienen como objetivo mejorar la estabilidad del vehículo. Sin embargo, estos vehículos permiten mejorar no solo el rendimiento dinámico, sino también la eficiencia, que es uno de los principales desafíos para los vehículos eléctricos, con el objetivo de aumentar su autonomía. En este trabajo, se ha propuesto un novedoso sistema de TV, compuesto por un estimador neuro-borroso para las fuerzas verticales en los neumáticos, un controlador basado en lógica borrosa y con capacidad de frenado regenerativo, siendo este último subsistema la principal contribución de este trabajo.

El sistema de TV propuesto se ha validado en el simulador de dinámica vehicular Dynacar en dos escenarios: una maniobra de emergencia y una vuelta en tres circuitos profesionales. Los resultados muestran que el sistema de TV desarrollado con la novedosa contribución de frenado regenerativo no solo es capaz de mejorar el rendimiento dinámico del vehículo, sino también la eficiencia general del vehículo, reduciendo la energía total consumida.

El trabajo futuro incluirá un estudio más sofisticado para las condiciones de prueba, incluidas más maniobras, a distintas velocidades, a fin de analizar el efecto del algoritmo de TV en todo el rango de velocidad de un automóvil.

\section{Agradecimientos}

Los autores desean agradecer al proyecto ECSEL HIPERFORM (con número de concesión 783174) y al proyecto H2020 ACHILES Project (con número de concesión 824311) por financiar el desarrollo de este trabajo. 


\section{English summary}

\section{A NOVEL TORQUE VECTORING ALGORITHM WITH REGENERA- TIVE BRAKING CAPABILITIES}

\begin{abstract}
Intelligent Transportation Systems (ITS) is currently one of the most active research areas, being electric vehicles (EVs) and their vehicle dynamics enhancement key topics. For this purpose, the development of optimal Advanced Driver-Assistance Systems (ADAS) and Advanced Vehicle Dynamics Control Systems (AVDC) is required. Conventionally, these systems have been focused on increasing the stability of the vehicle in critical scenarios. However, EVs enable the possibility of including also the efficiency by making use of the regenerative braking as a control variable. In order to be able to design such sophisticated control systems, it is necessary to implement control techniques capable to manage both stability and efficiency. In this sense, intelligent control techniques have demonstrated to be one of the best alternatives. In this work a Torque Vectoring (TV) algorithm based on intelligent control techniques and with regenerative braking capabilities is presented. The presented TV approach has been implemented in a embedded platform and tested in a Hardware in the Loop (HiL) setup. Results show that the presented approach is able to not only enhance the dynamics vehicle behaviour in a challenging emergency manoeuvre, but also to increase its overall efficiency.
\end{abstract}

Keywords: Electric vehicle, torque vectoring, vehicle dynamics, regenerative braking.

\section{Referencias}

[1] Cuadrado, J., Vilela, D., Iglesias, I., Martín, A., Peña, A., (2013) "A Multibody Model to Assess the Effect of Automotive Motor Inwheel Configuration on Vehicle Stability and
Comfort", ECCOMAS Multibody Dynamics 2013.

[2] Dendaluce, M., Iglesias, I., Martín, A., Prieto, P., Peña, A., (2016) "Race-Track testing of a Torque Vectoring Algorithm on a Motorin-Wheel Car using a Model-Based Methodology with a HiL and Multibody Simulator Setup", International Conference on Intelligent Transportation Systems.

[3] De Novellis, L., Sorniotti, A., Gruber, P., Pennycot, A., (2014) "Comparison of Feedback Control Techniques for TorqueVectoring Control of Fully Electric Vehicles" IEEE Transactions on Vehicular Technology.

[4] "Dynacar by Tecnalia." [Online]. Available: http://www.dynacar.es/en/home.php.

[5] Pacejka, H.B. "Tyre and Vehicle Dynamics", BH, 2005.

[6] Shimada, K., Shibahata, Y. (1994), "Comparison of Three Active Chassis Control Methods for Stabilizing Yaw Moments", $S A E$ Technical Paper.

[7] Wallentowitz, H., (2005) "Vertical and Lateral Dynamics of Passenger Vehicles", Automotive Technology II, Institute of Automotive Engineering, Aachen University of Technology, Germany.

[8] Zadeh, L.. (1965) 'Fuzzy Sets" Information and Control.

[9] ISO 3888-2:2011 Passenger cars - Test track for a severe lane-change manoeuvre - Part 2: Obstacle avoidance.

[10] European Roadmap Electrification of Road Transport, ERTRAC.

[11] S. Ding, L. Liu and W. X. Zheng, "Sliding Mode Direct Yaw-Moment Control Design for In-Wheel Electric Vehicles," in IEEE Transactions on Industrial Electronics, vol. 64, no. 8, pp. 6752-6762, Aug. 2017.

[12] Alberto Parra, Asier Zubizarreta, Joshué Pérez, and Martín Dendaluce, "Intelligent Torque Vectoring Approach for Electric Vehicles with Per-Wheel Motors," Complexity, vol. 2018.

[13] G. Kaiser, Q. Liu, C. Hoffmann, M. Korte, H. Werner, "LPV Torque Vectoring for an Electric Vehicle with Experimental Validation", IFAC Proceedings Volumes, Volume 47, Issue 3, 2014, Pages 12010-12015. 
[14] H. Guo, F. Liu, F. Xu, H. Chen, D. Cao and Y. Ji, "Nonlinear Model Predictive Lateral Stability Control of Active Chassis for Intelligent Vehicles and Its FPGA Implementation," in IEEE Transactions on Systems, Man, and Cybernetics: Systems, vol. PP, no. 99, pp. 1-12.

[15] L. Yue-Lin, H. Ping-Wen and X. Tao, "A Research on Adaptive Neural Network Control Strategy of Vehicle Yaw Stability," 2013 Fourth International Conference on Intelligent Systems Design and Engineering Applications, Zhangjiajie, 2013, pp. 48-51.

[16] J. Kim, H. Lee, and S. Choi, "A robust road bank angle estimation based on a proportional-integral Hfilter," Proc. Inst. Mech. Eng. D, J. Autom. Eng., vol. 226, no. 6, pp. 779-794, Jun. 2012.

[17] R. Ghandour, A. Victorino, M. Doumiati, and A. Charara, "Tire/road friction coefficient estimation applied to road safety," in Proc. 18th Mediterranean Conf. Control Autom. Congr., 2010, pp. 1485-1490.

[18] De Novellis L, Sorniotti A, Gruber P. Driving modes for designing the cornering response of fully electric vehicles with multiple motors.Mech Syst Signal Process. 2015;64:1-15.

[19] Lenzo B, Sorniotti A, De Filippis G, et al. Understeer characteristics for energy-efficient fully electric vehicles with multiple motors. In: EVS29 international battery, hybrid and fuel cell electric vehicle symposium proceedings; 2016.

[20] De Filippis G, Lenzo B, Sorniotti A, et al. Energy-efficient torque-vectoring control of electric vehicles with multiple drivetrains. IEEE Trans Veh Technol. 2018;67(6):4702-4715.

[21] Tota A, Lenzo B, Lu Q, et al. On the experimental analysis of integral sliding modes for yaw rate and sideslip control of an electric vehicle with multiple motors. Int J Automot Technol.

[22] Chindamo D, Lenzo B, Gadola M. On the vehicle sideslip angle estimation: a literature review of methods, models, and innovations. Appl Sci. 2018;8(3):355.

[23] Frendo F, Greco G, Guiggiani M. Critical review of handling diagram and understeer gradient for vehicles with locked differential. Veh Syst Dyn.
[24] Frendo F, Greco G, Guiggiani M, et al. The handling surface: a new perspective in vehicle dynamics. Veh Syst Dyn.

[25] Bucchi F, Frendo F. A new formulation of the understeer coefficient to relate yaw torque and vehicle handling. Veh Syst Dyn.

[26] Edelmann J, Plochl M. Analysis of controllability of automobiles at steady-state cornering considering different drive concepts. Proceedings of IAVSD 2017 (Dynamics of Vehicles on Roads and Tracks); 2017.

[27] Acosta, M., Kanarachos, S. Neural Comput Applic (2017). https://doi.org/10.1007/s00521-017-2932-9

[28] https://www.fsaeonline.com/

[29] V. Ivanov, D. Savitski and B. Shyrokau, "A Survey of Traction Control and Antilock Braking Systems of Full Electric Vehicles With Individually Controlled Electric Motors," in IEEE Transactions on Vehicular Technology, vol. 64, no. 9, pp. 3878-3896, Sept. 2015.

[30] Basilio Lenzo, Francesco Bucchi, Aldo Sorniotti and Francesco Frendo (2018) On the handling performance of a vehicle with different front-to-rear wheel torque distributions, Vehicle System Dynamics.

[31] Sakawa, Masatoshi, "Genetic Algorithms and Fuzzy Multiobjective Optimization", Springer, 2002.

[32] Kakalis L., Cheli F., Sabbioni E.: "The development of a brake based torque vectoring system for a sport vehicle performance improvement", Proc. of 6th International Conference on Informatics in Control, Automation and Robotics (ICINCO 2009), Vol 1 ICSO, pp. 298-304, Milano, Italy, 2-5 July, 2009.

[33] Sabbioni, E., Kakalis, L., and Cheli, F., "On the Impact of the Maximum Available Tire-Road Friction Coefficient Awareness in a Brake-Based Torque Vectoring System," SAE Technical Paper 2010-01-0116, 2010, doi:10.4271/2010-01-0116.

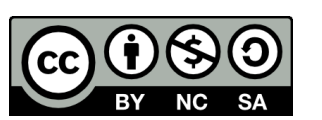
(C) 2019 by the authors. Submitted for possible open access publication under the terms and conditions of the Creative Commons Attribution CC BY-NC-SA 4.0 license (https://creativecommons.org/licenses/by-ncsa/4.0/deed.es). 